\title{
DIVERSITÉ FLORISTIQUE DES SUBERAIES DU PARC NATIONAL DE TLEMCEN (ALGERIE)
}

\author{
Assia LETREUCH-BELAROUCI* ${ }^{* 1}$, Boumediene MEDJAHDI ${ }^{1}$, \\ Noureddine LETREUCH-BELAROUCI ${ }^{1} \&$ Kheloufi BENABDELI ${ }^{2}$ \\ ${ }^{1}$ Département de Foresterie, Faculté des Sciences, Université de Tlemcen. \\ B.P 12 IMAMA. TLEMCEN 13000. ALGERIE \\ ${ }^{2}$ Département de Biologie, Université de Mascara Algérie \\ *Corresponding author: letreuch_assia@yahoo.com
}

Recibido el 9 de marzo de 2009, aceptado para su publicación el 20 de junio de 2009

Publicado "on line" en julio de 2009

RESUME. Diversité floristique des suberaies du Parc National de Tlemcen (Algérie). L'analyse de la diversité floristique des forêts de Hafir Zariffet a révélé l'existence de 211 taxons appartenant à 65 familles et 164 genres. Le nombre de taxons endémiques et/ou rares inventoriés comporte: 1 endémique algérien, 7 algéro-marocains, 11 ibéro-algéro-marocains, 7 sont endémiques d'Afrique du Nord et 11 sont des endémiques d'Afrique du Nord et de la Péninsule ibérique. Le nombre des taxons rares s'élève à 26 dont 9 sont en même temps rares est menacés. Des efforts urgents de protection doivent êtres consentis pour préserver le chêne liège ainsi que les espèces endémiques qui lui sont inféodées.

Mots clés. Subéraies, inventaire, diversité, Parc National de Tlemcen, Algérie.

SUMMARY. Flora diversity of cork oak forest in the National Park of Tlemcen (Algeria). The analysis of the floristic diversity of forest massif "Hafir-Zariffet" in the Tlemcen National Park has revealed the existence of 211 taxa which belong to 65 families and 164 genera. The number of taxa endemic and/or rare includes: 1 endemic algerian, 7 algero-moroccan, 11 ibero-algero-moroccan, 7 are endemic in North Africa and 11 are endemic in Iberian Peninsula and North Africa. The number of rare taxa is 26, 9 are at the same time threatened. Urgent efforts are required to preserve the cork oak as well as endemic species confined to it.

Key words. Cork oak forest, inventory, diversity, National Park of Tlemcen, Algeria.

\section{INTRODUCTION}

Comme région naturelle assez singulière par sa diversité et ses richesses, les monts de
Tlemcen ont toujours intéressé les chercheurs. Ce domaine montagneux, malgré la pression anthropo-zoogène, reste une région «forestière» par excellence même si la végétation se présente 
sous forme de matorrals à différents états de dégradation (Letreuch, 2002). Dans tout cet ensemble montagneux, s'inscrit sur 8200 ha le «Parc National de Tlemcen».

C'est un grand territoire aux multiples facettes écologiques. On y distingue principalement deux étages bioclimatiques: sub-humide et semi-aride, ce qui lui confère une position tant géographique qu'orographique particulière formant de grandes zones de végétation s'étendant successivement de la forêt feuillue, au sud ouest, en passant par la chênaie mixte au nord puis aux forêts artificielles résineuses et aux matorrals de chêne vert naturel au nord-est. Les reliefs présentent de grands ensembles bien tranchés, comme les plaines et les vallées situées sur des terrains tertiaires et quaternaires, alors que les hauts massifs forestiers montagneux sont assis sur des terrains du jurassique.

Le travail de recherche entrepris dans cet article concerne essentiellement les subéraies des forêts de Hafir et de Zariffet qui constituent une des très rares formations naturelles reliques de chêne liège de montagne de l'Ouest de
l'Algérie. En raison de l'insuffisance d'études floristiques sur ce massif très accidenté, un échantillonnage a été réalisé ce qui nous a permis d'établir un catalogue actualisé de la région avec des indications particulières aux espèces rares, menacées et/ ou endémiques.

\section{Présentation de la zone d'étude}

L'étude de la diversité floristique s'est déroulée dans les suberaies de la partie Nord des monts de Tlemcen où se trouve un groupement comportant les deux intéressants massifs de chêne liège de Zariffet (962 ha) et d'Hafir (9.872 ha). Le climat est marqué par une sécheresse estivale qui se manifeste dès le mois de juin $(7,2$ $\%$ seulement de précipitations estivales, sur une tranche annuelle de $483 \mathrm{~mm}$ ). La moyenne des températures maximales du mois le plus chaud (M) est de $32,35^{\circ} \mathrm{C}$, celle des minima du mois le plus froid (m) est de $3,2^{\circ} \mathrm{C}$. L'indice pluviométrique d'Emberger est de 51,1, ce qui confirme un climat semi-aride supérieur à variante tempérée. L'altitude varie de 800 à $1418 \mathrm{~m}$ et imprime au relief accidenté toutes les expositions (Parc National Tlemcen, 2000).

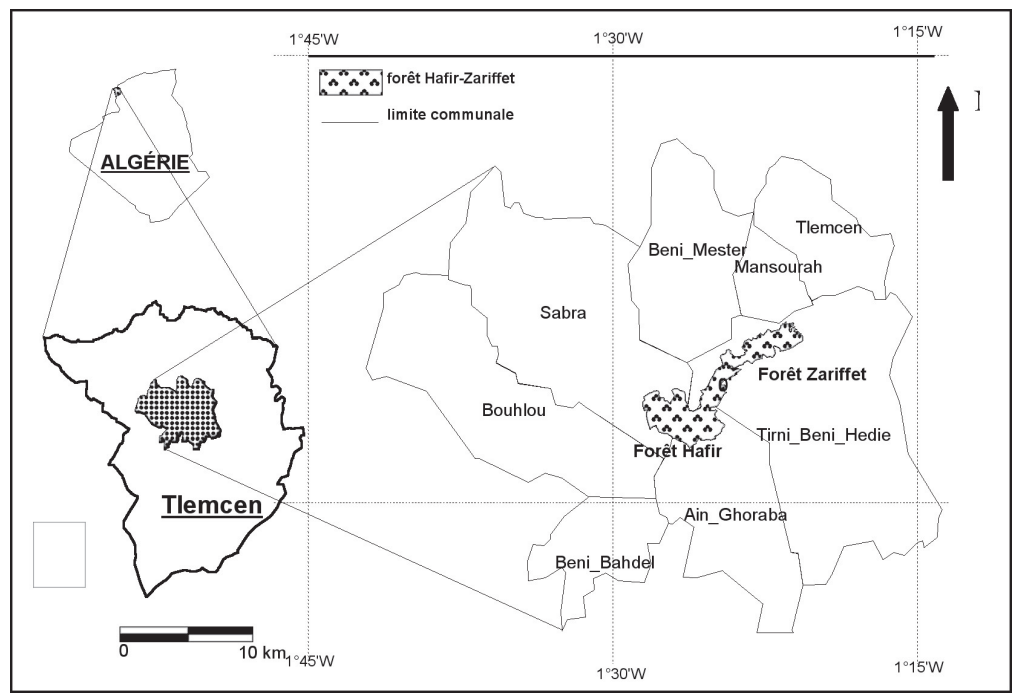

Figure 1. Carte de localisation du massif Hafir-Zariffet constituant la zone intégrale du Parc National de Tlemcen depuis 1993 (Letreuch, 2002) 
Les deux forêts reposent sur un massif datant du jurassique supérieur constitué dans sa majorité de grès séquanien et d'alluvions quaternaires. Les sols sont en général plus ou moins profonds de type brun forestier, la strate herbacée est assez riche d'où l'existence d'une forte activité biologique. La texture est sablo-limoneuse avec quelques concrétions à l'horizon A1. L'humus est bien abondant et les matières organiques sont importantes. Les sols bruns fersiallitiques sont également très développés (Gaouar, 1980).

Le massif est composé essentiellement de peuplements naturels de chêne liège. On n'aperçoit que de rares traces de régénération naturelle, malgré l'existence d'un sol profond (Letreuch, 1991). L'importance des pressions et l'absence de gestion adéquate sont à l'origine de cet état. Le reste du massif est occupé par des peuplements de chêne vert et de chêne zéen.

Les relevés phytosociologiques effectués par Zeraia (1981) et Dahmani (1984), au niveau de ces forêts ont permis de les rattacher au Cytiso triflori-Quercetum suberis $\mathrm{Br} . \mathrm{Bl}$ sousassociation Quercetosum canariensis. La physionomie de la végétation la plus évoluée est celle d'un matorral arboré élevé et dense.

\section{MATERIEL ET METHODES}

La réalisation du présent catalogue a été faite à partir de travaux de terrain de l'année 2004 à 2008. Les échantillons témoins sont déposés dans l'herbier du laboratoire Gestion Conservatoire de l'Eau, du sol et des forêts de l'université de Tlemcen.

Nous avons procédé par la suite à la détermination des espèces récoltées. Les ouvrages de base utilisés sont les flores de l'Algérie et du Maroc (Quézel et Santa (1962), Fennane et al. (1999 \& 2007) et Valdes et al. (2002)). Nous avons d'autre part sélectionné les espèces rares, menacées ou endémiques présentes dans les relevés.
Les informations données dans ce catalogue se présentent dans l'ordre suivant:

Nom scientifique: La nomenclature adoptée est celle de la Flore d'Algérie ou à défaut de la Flore pratique du Maroc (Fennane et al. (eds.), 1999) et du Catalogue des Plantes vasculaires du Nord du Maroc de Valdés et al. (eds.) (2002).

Type biologique: $\mathrm{Ph}$. (Phanérophyte), Nph. (Nanophanérophyte), Ch. (Chaméphyte), H. (Hémicryptophyte), G. (Géophyte), Th. (Thérophyte), Phl. (Phanérophyte liane).

Répartition géographique: Pour chaque taxon la répartition biogéographique en Algérie est indiquée pour les divisions adoptées par Quézel et Santa (1962):

01: Sahels littoraux oranais 02: plaines littorales oranaises. O3: l'Atlas tellien oranais. A1: littoral algérois. A2: 1'Atlas tellien algérois. K1: la Grande Kabylie K2: la Petite Kabylie. K3: la Numidie. C1: Tell constantinois. H1: Hauts-plateaux algérois et oranais. H2: Hauts plateaux constantinois. Hd: Hodna. AS1: 1'Atlas saharien oranais. AS2: 1'Atlas saharien algérois. AS3: l'Atlas saharien constantinois. SS1: Sahara septentrional occidental. SS2: Sahara septentrional oriental. SO: Sahara Occidental. SC: Sahara Central. SM : Sahara méridional.

Espèces endémiques: Nous distinguons les endémiques de l'Algérie [A]; de l'Algérie et du Maroc [AM]; de l'Algérie, du Maroc et de la Péninsule Ibérique [AMI]; de l'Algérie, du Maroc et de la Tunisie [Afn] et enfin de l'Afrique du Nord et de la Péninsule Ibérique [AfnI].

Espèces rares: Afin d'avoir une idée sur l'abondance globale de chaque taxon dans l'ensemble du territoire algérien, nous avons ajouté le degré d'abondance adopté par Quézel et Santa (1962). Cet indice possède huit niveaux allant de " extrêmement rare » (RRR) à «extrêmement commun» (CCC). Seuls cinq niveaux seront retenus: les trois premiers correspondent à une rareté plus ou moins grande : $\mathrm{RR}$ : très rare; $\mathrm{R}$ : rare et les trois autres liés au degré d'abondance: AC: Assez 
commun; C: Commun, CC: Très commun. AS1-2-3) AC.

\section{RESULTATS \\ PTERIDOPHYTES \\ ADIANTACEAE}

Adiantum capillus-veneris $\mathrm{L}$.

G., (O1-2-3, A1-2, K1-2-3, C1 et AS1-2-3), C.

\section{ASPLENIACEAE}

Asplenium adiantum-nigrum $\mathrm{L}$.

G., (O1-2-3, A1-2, K1-2-3, C1 et AS1-2-3), AC.

Asplenium ceterach $\mathrm{L}$.

G., (O1-2-3, A1-2, K1-2-3, C1 et AS1-2-3), AC.

Asplenium trichomanes L.

G., (O1-2-3, A1-2, K1-2-3, C1 et AS1-2-3), AC.

\section{EQUISETACEAE}

Equisetum ramosissimum Desf.

G.(NPh)., (O1-2-3, A1-2, K1-2-3, C1, H1-2, Hd et AS1-2-3), AC.

\section{SELAGINALLACEAE}

Selaginella denticulata (L.) Spring

H., (O1-2-3, A1-2, K1-2-3 et C1), C.

\section{SINOPTERIDACEAE}

Cheilanthes acrostica (Balb.) Tod.

G., (O1-2-3, A1-2, K1-2-3, C1, H1-2, Hd, AS12-3 et SS), C.

Cosentinia vellea (Aiton) Tod.

H., (O1-2-3, A1-2, K1-2-3, C1 et AS1-2-3), AC.

\section{WOODSIACEAE}

Cystopteris fragilis (L.) Bernh.

G., (K1-2-3, C1, A2 et AS3), R.

GYMOSPERMES

CUPRESSACEAE

Juniperus oxycedrus L.

Ph., (O1-2-3, A1-2, K1-2-3, C1, H1-2, Hd et
PINACEAE

Pinus halepensis Mill.

Ph., (O1-2-3, A1-2, K1-2-3, C1, H1-2, Hd et AS1-2-3), C.

\section{DICOTYLEDONES}

ANACARDIACEAE

Pistacia lentiscus $\mathrm{L}$.

Nph., (O1-2-3, A1-2, K1-2-3, C1, H1-2, Hd et AS1-2-3), C.

\section{APOCYNACEAE}

Nerium oleander L.

Ph., (O1-2-3, A1-2, K1-2-3, C1, H1-2, Hd et AS1-2-3), C.

\section{ARALIACEAE}

Hedera helix auct., non L.

Phl., (O1-2-3, A1-2, K1-2-3 et C1), C.

\section{ARISTOLOCHIACEAE}

Aristolochia baetica L.

Phl., (O1-2-3), [AMI], C.

\section{ASTERACEAE}

Anacyclus clavatus (Desf.) Pers.

Th., (O1-2-3, A1-2, K1-2-3, C1, H1-2, Hd et AS1-2-3), C.

Andryala integrifolia $\mathrm{L}$.

H., (O1-2-3, A1-2, K1-2-3, C1, H1-2, Hd et AS1-2-3), C.

Bellis sylvestris Cirillo

H., (O1-2-3, A1-2, K1-2-3 et C1), C.

Bombycilaena discolor (Pers.) M. Lainz

Th., (O1-2-3, A1-2, K1-2-3, C1, H1-2, Hd et AS1-2-3), C.

Calendula arvensis $\mathrm{L}$.

Th., (O1-2-3, A1-2, K1-2-3, C1, H1-2, Hd et AS1-2-3), C.

Catananche caerulea $\mathrm{L}$.

H., (O1-2-3, A1-2, K1-2-3 et C1), C.

Catananche caespitosa Desf. 
H., (C1, O3, H1-2 et AS3), AR.

Catananche lutea $\mathrm{L}$.

Th., (O1-2-3, A1-2, K1-2-3, C1, H1-2, Hd et AS1-2-3), C.

Carduus pycnocephalus L.

Th., (O1-2-3, A1-2, K1-2-3 et C1), C.

Carthamus lanatus L.

Th., (O1-2-3, A1-2, K1-2-3 et C1), C. Coleostephus multicaulis (Desf.) Durieu

Th., (O1-2-3 et H1), [A], C.

Dittrichia viscosa (L.) Greuter

Ch., (O1-2-3, A1-2, K1-2-3 et C1), C.

Echinops strigosus L.

Th., (O1-2-3), [AfnI], C.

Evax argentea Pomel

Th., (O2, AS3, A2 et SS), R.

Hedypnois rhagadioloides (L.) F.W. Schmidt

Th., (O1-2-3, A1-2, K1-2-3 et C1), C.

Heteromera fuscata (Desf.) Pomel

Th., (AS, H1-2, SS C1), R.

Hieracium amplexicaule $L$.

H., (O3), RR.

Hypochaeris glabra L.

Th., (O1-2-3, A1-2, K1-2-3, C1, AS1-2-3, H1-2 et $\mathrm{Hd}), \mathrm{AC}$.

Filago pyramidata $\mathrm{L}$.

Th., (O1-2-3, A1-2, K1-2-3, C1, H1-2, Hd et AS1-2-3), C.

Leontodon hispidulus (Delile) Boiss.

H., (O1-2- 3, A1-2, K1-2-3, C1, H1-2, Hd et AS1-2-3), [AfnI], C.

Mauranthemum paludosum (Poir.) Vogt et Oberprieler

Th., (O1-2-3, A1-2, K1-2-3 et C1), [AfnI], C.

Pallenis spinosa (L.) Cass.

H., (O1-2-3, A1-2, K1- 2-3, C1, H1-2, Hd et AS1-2-3), C.

Pulicaria odora (L.) Rchb.

H., (O1-2-3, A1-2, K1- 2-3, C1, H1-2, Hd et AS1-2-3), C.

Senecio vulgaris $\mathrm{L}$.

Th., (O1-2-3, A1-2, K1-2-3, C1, H1-2, Hd et AS1-2-3), C.

Urospermum picroides (L.) Scop. ex F.W. Schmidt

Th., (O1-2-3, A1-2, K1-2-3, C1), C.

BORAGINACEAE

Cynoglossum cheirifolium $\mathrm{L}$.
Th., (O1-2-3, A1-2, K1-2-3, C1, H1-2, Hd et AS1-2-3), C.

Echium horridum Batt.

H., (AS1-2-3, SS et SC), AR.

Neatostema apulum (L.) I. M.

Th., (O1-2-3, A1-2, K1-2-3, C1, H1-2, Hd et AS1-2-3), AC.

\section{BRASSICACEAE}

Biscutella baetica boiss. \& Reut.

Th., (O1-2-3, A1-2, K1-2-3, C1, H1-2, Hd, AS12-3 et SS1-2), [AMI], C.

Carrichtera annua (L.) DC.

Th., (O1-2-3, A1-2, K1-2-3, C1, H1-2, Hd et AS1-2-3), C.

Eruca setulosa Boiss.\& Reuter

H., (O3), [AM.], RR.

Lepidium draba $L$.

H., (O1-2-3, A1-2, K1-2-3, C1, H1-2, Hd et AS1-2-3), AR.

Lobularia maritima (L.) Desv.

Ch., (O1-2-3, A1-2, K1-2-3, C1, H1-2, Hd et AS1-2-3), C.

Raphanus raphanistrum $L$

Th., (O1-2-3, A1-2, K1-2-3, C1, H1-2, Hd et AS1-2-3), AC.

\section{CAMPANULACEAE}

Campanula trachelium subsp. mauritanica (Pomel) Quézel AC.

H., (O1-2-3, A1-2, K1-2-3, C1, Hd et AS3),

\section{CAPRIFOLIACEAE}

Lonicera implexa $\mathrm{L}$.

Phl., (O1-2-3, A1-2, K1-2-3 et C1), AC.

Viburnum tinus $\mathrm{L}$.

Ph., (O1-2-3, A1-2, K1-2-3 et C1), AC.

\section{CARYOPHYLLACEAE}

Cerastium brachypetalum Pers.

Th., (O1-2-3, A1-2, K1-2-3, C1 et AS1-2-3), AC.

Pteranthus dichotomus Forssk.

Th., (O2-3, H1-2, SS et SC), AC.

Silene colorata subsp. trichocalycina Fenzl 
Th., (O1-2-3), C.

Silene secundiflora Otth

Th., (O1-2-3, A1-2, K1-2-3, C1, AS1-2-3, H1-2 et $\mathrm{Hd}$.), $\mathrm{R}$.

\section{CISTACEAE}

Cistus creticus L.

Ch.(NPh.), (O1-3, A1-2 et AS1), AC.

Cistus ladaniferus L.

NPh., (O1-3 et A1-2), C.

Cistus monspeliensis $\mathrm{L}$.

NPh., (O1-2-3, A1-2, K1-2-3, C1, H1-2, Hd, AS1-2-3), C.

Cistus salviifolius $\mathrm{L}$.

Ch.(NPh.), (O1-2-3, A1-2, K1-2-3 et C1), AC.

Fumana laevipes (L.) Spaech

Ch., (O1-2-3, A1-2, K1-2-3, C1, H1-2, Hd et AS1-2-3), C.

Fumana thymifolia (L.) Spach ex Webb

Ch., (O1-2-3, A1-2, K1-2-3, C1, H1-2, Hd et AS1-2-3), C.

Halimium umbellatum subsp. viscosum Willk.

Ch., (O3), [AMI], R.

Helianthemum origanifolium subsp. molle (Cav.) Font Quer \& Rothm.

Ch., (O1), [AMI] R.

Helianthemum viscarum Boiss. \& Reut.

Ch., (O1-2-3), [AMI], AC.

Tuberaria guttata (L.) Fourr.

Th., (O1-2-3, A1-2, K1-2-3 et C1), AC.

\section{CONVOLVULACEAE}

Convolvulus althaeoides $\mathrm{L}$.

H., (O1-2-3, A1-2, K1-2-3, C1, H1-2, Hd et AS1-2-3), C.

Convolvulus cantabrica $\mathrm{L}$.

H., (O1-2-3, A1-2, K1-2-3 et C1), C.

\section{CRASSULACEAE}

Sedum sediforme (Jacq.) Pau

Ch., (O1-2-3 et K1-2-3), C.

Umbilicus rupestris (Salisb.) Dandy

H., (O1-2-3, A1-2, K1-2-3 et C1), C.

\section{DIPSACACEAE}

Cephalaria leucantha (L.) Roem. \& Schult.
H., (A2 et O3), $\mathrm{R}$.

Scabiosa atropurpurea $\mathrm{L}$.

Th.(H.), (O1-2-3, A1-2, K1-2-3, C1, H1-2, Hd et AS1-2-3), C.

Scabiosa stella $\mathrm{L}$.

Th., (O1-2-3, A1-2, K1-2-3, C1, H1-2, Hd et AS1-2-3), C.

\section{ERICACEAE}

Arbutus unedo L.

NPh., (O1-2-3, A1-2, K1-2-3, C1 et AS1-2), C.

Erica arborea $\mathrm{L}$.

NPh., (O1-2-3, A1-2, K1-2-3, C1, AS1-3), C.

\section{EUPHORBIACEAE}

Euphorbia helioscopia L.

Th., (O1-2-3, A1-2, K1-2-3, C1, AS1-3), C.

Euphorbia terracina $\mathrm{L}$.

Th., (O1-2-3, A1-2, K1-2-3, C1, H1-2, Hd et AS1-2-3), C.

\section{FAGACEAE}

Quercus faginea subsp. tlemcenensis (A. DC.) Greuter \& Burdet

Ph., (O3), [AM], AC.

Quercus coccifera $\mathrm{L}$.

NPh.(Ph), (O1-2-3, A1-2, K1-2-3, C1et AS3), C.

Quercus rotundifolia Lam.

Ph., (O1-2-3, A1-2, K1-2-3, C1), AC.

Quercus suber L.

Ph., (O1-3, A1-2, K1-2-3 et C1), AC.

\section{FUMARIACEAE}

Fumaria capreolatea L.

Th., (O1-2-3, A1-2, K1-2-3, C1, H1-2, Hd et AS1-2-3), C.

Fumaria densiflora DC.

Th., (O1-2-3, A1-2, K1-2-3 et AS1-2-3), C.

Fumaria parviflora Lam.

Th., (O1-2-3, A1-2, K1-2-3, C1, H1-2, Hd et AS1-2-3), C.

\section{GENTIANACEAE}

Blackstonia perfoliata subsp. perfoliata 
Th., (K2-3), R.

Centaurium erythraea Rafin.

H.(Th.), (O1-2-3, A1-2, K1-2-3 et C1), C.

\section{GERANIACEAE}

Geranium purpureum Vill.

Th.(H.), (O1-2-3, A1-2, K1-2-3, C1, H1-2, Hd et AS1-2-3), C.

\section{LAMIACEAE}

Ajuga iva (L.) Schreb.

H.(Th.), (O1-2-3, A1-2, K1-2-3 et C1), C.

Lamium amplexicaule $\mathrm{L}$.

Th., (O1-2-3, A1-2, K1- 2-3, C1, H1-2, Hd et AS1-2-3), C.

Lavandula stoechas $\mathrm{L}$.

Ch., (O1-2-3, A1-2, K1-2-3 et C1), C.

Marrubium vulgare $\mathrm{L}$.

Ch., (O1-2-3, A1-2, K1-2-3, C1, H1-2, Hd et AS1-2-3), C.

Prasium majus L.

NPh., (O1-2-3, A1-2, K1-2-3, C1, H1-2, Hd et AS1-2-3), C.

Salvia algeriensis Desf.

Th., (O1-2-3), C.

Salvia verbenaca $\mathrm{L}$.

H., (O1-2-3, A1-2, K1-2-3, C1, H1-2, Hd et AS1-2-3), C.

Salvia viridis $\mathrm{L}$.

H., (O1-2-3, A1-2, K1-2-3 et C1), AC.

Teucrium capitatum $\mathrm{L}$.

Ch., (O1-2-3, A1-2, K1-2-3, C1, H1-2, Hd et AS1-2-3), C.

Teucrium fruticans $\mathrm{L}$.

Ch., (O1-3 et H1), AC.

Teucrium pseudo-champaepytis $\mathrm{L}$.

Ch., (O1-2-3, A1-2, K1-2-3 et C1), C.

Thymus munbyanus subsp. coloratus (Boiss. \& Reut.) Greuter \& Burdet

Ch., (O1-2-3, A1-2,K1-2-3 et C1), [AM], AC.

Thymus munbyanus subsp. munbyanus

Ch. (O1-2- 3 et H1-2), [AM], AC.

\section{LEGUMINOSAE}

Anthyllis vulneraria subsp. saharae (Sagorski) Jahand. \& Maire

Th.(H.), (O1-2-3, A1-2, K1-2-3 et C1), [Afn.], C.
Calicotome intermedia $\mathrm{C}$. Presl.

$\mathbf{N P h},,(\mathrm{O} 1-2-3), \mathrm{C}$.

Ceratonia siliqua $\mathrm{L}$.

Ph., (O1-2-3, A1-2, K1-2-3, C1 et AS1-2-3), C.

Coronilla scorpioides (L.) W.D.J. Koch

Th., (O1-2-3, A1-2, K1-2-3, C1, H1-2, Hd et AS1-2-3), C.

Cytisus arboreus (Desf.) DC.

NPh., (O1-2-3), RR.

Cytisus villosus Pourr.

NPh., (O1-3, A1-2, C1), R.

Genista tricuspidata Desf.

NPh., (O1-2-3, A1-2, K1-2-3, C1 et SS1), [Afn.], C.

Genista ramosissima (Desf.) Poir.

NPh., (O1-2-3), AC.

Hippocrepis ciliata Willd.

Th., (O1-2-3, A1-2, K1-2-3, C1, H1-2, Hd et AS1-2-3), C.

Hippocrepis multisilliquosa $\mathrm{L}$.

Th., (O1-2-3, A1-2, K1-2-3, C1, AS1-2-3 et SS1-2), C.

Lupinus hirsutus $\mathrm{L}$.

Th., (O1-2-3, A1-2, K1-2-3 et C1), AC.

Medicago murex Willd.

Th., (A1-2, C1), AC.

Medicago truncatula Gaertn.

Th., (O1-2-3, A1-2, K1-2-3 et C1), C.

Ononis spinosa $\mathrm{L}$.

Ch., (O1-2-3 et A2), R.

Scorpiurus sulcatus L.

Th., (O1-2-3, A1-2, K1-2-3 et C1), C.

Trifolium angustifolium $\mathrm{L}$.

Th., (O1-2-3, A1-2, K1-2-3, C1 et AS3), C.

Trifolium arvense $\mathrm{L}$.

Th., (O1-2-3, A1-2, K1-2-3, C1 et AS3), C.

Trifolium campestre Schreb.

Th., (O1-2-3, A1-2, K1-2-3, C1 et AS1-2-3), C.

Trifolium cherleri $\mathrm{L}$.

Th., (O1-2-3, A1-2, K1-2-3, C1 et AS1-2-3), C.

Trifolium squarrosum $\mathrm{L}$.

Th., (O1-2-3, A1-2, K1- 2-3 et C1), C.

Tripodion tetraphyllum (L.) Four.

Th., (O1-2-3, A1-2, K1-2-3 et C1), C.

Ulex boivinii Webb

NPh., (O3), [AMI], R.

\section{LINACEAE}

Linum austriacum subsp. mauritanicum (Pomel) 
Greuter \& Burdet

Th. (H1-2) AC.

Linum strictum $\mathrm{L}$.

Th., (O1-2-3, A1-2, K1-2-3, C1, H1-2, Hd et AS1-2-3), AC.

Linum usitatissimum $\mathrm{L}$.

Ch., (O1-2-3, A1-2, K1-2-3, C1, H1-2, Hd et AS1-2-3), AC.

\section{MALVACEAE}

Malva sylvestris $\mathrm{L}$.

H., (O1-2-3, A1-2, K1-2-3, C1, H1-2, Hd, AS12-3, SS1-2), C.

OLEACEAE

Olea europea $\mathrm{L}$.

Ph., (O1-2-3, A1-2, K1-2-3, C1, AS1-2-3 et SS1-2), C.

Phillyrea angustifolia $\mathrm{L}$.

NPh., (O1-3, A2, K1-2-3, C1, AS1-2-3), C.

\section{OROBANCHACEAE}

Orobanche minor $\mathrm{Sm}$.

G., (O1-2-3, A1-2, K1-2-3, C1, H1-2, Hd, AS12-3 et SS1-2, SC), C.

Orobanche variegata Wallr.

G., (O1-2-3, A1-2, K1-2-3, C1, H1-2 et Hd), C.

\section{PAPAVERACEAE}

\section{Glaucium flavum Crantz}

H., (O1-2, A1, K1-2-3), AC.

Papaver rhoeas L.

Th., (O1-2-3, A1-2, K1-2-3, C1, H1-2, Hd et AS1-2-3), C.

\section{PLANTAGINACEAE}

\section{Plantago afra $\mathrm{L}$.}

Th., (O1-2-3, A1-2, K1-2-3, C1, H1-2, Hd, AS12-3, SS1-2 et SC), C.

Plantago cupanii Guss.

Th.(H.), (O1-2-3, A1-2, K1-2-3 et C1), AC.

Plantago lagopus $\mathrm{L}$.

Th., (O1-2-3, A1-2, K1-2-3, C1, H1-2, Hd et AS1-2-3), C.

Plantago lanceolata $\mathrm{L}$.

H., (O1-2-3, A1-2, K1-2-3, C1, H1-2, Hd et AS1-2-3), C.

\section{POLYGALACEAE}

Polygala monspeliaca L.

Th., (O1-2-3, A1-2, K1-2-3, C1, H1-2, Hd, AS1-2-3), C.

\section{POLYGONACEAE}

Rumex bucephalophorus L.

Th., (O1-2-3, A1-2, K1-2-3, C1 et AS1-2-3), C.

\section{PRIMULACEAE}

Anagallis arvensis subsp. latifolia (L.) Arcang.

Th., (O1-2-3, A1-2, K1-2-3 et C1), C.

Anagallis arvensis subsp. arvensis

Th., (O1-2-3, A1-2, K1-2-3, C1, H1-2, Hd, AS12-3 etSS1-2), C.

Asterolinon linum-stellatum (L.) Duby

Th., (O1-2-3, A1-2, K1-2-3, C1, H1-2, Hd et AS1-2-3), C.

\section{RANUNCULACEAE}

Adonis annua $\mathrm{L}$.

Th., (O1-2-3, A1-2, K1-2-3 et C1), AC.

Clematis vitalba $\mathrm{L}$.

Phl., (AS3), RR.

Ranuncula paludosus Poir.

H., (O1-2-3, A1-2, K1-2-3, C1, H1-2, Hd et AS1-2-3), C.

\section{RHAMNACEAE}

\section{Rhamnus alaternus L.}

Ph., (O1-2-3, A1-2, K1-2-3, C1, H1-2, Hd et AS1-2-3), C.

Rhamnus lycioides subsp. oleoides (L.) Maire

NPh., (O1-2-3, A1-2, K1-2-3, C1, H1-2, Hd, et AS1-2-3), AC.

\section{RESEDACEAE}

Reseda alba $\mathrm{L}$.

Th., (O1-2-3, A1-2, K1-2-3, C1, H1-2, Hd, AS12-3 et SS1-2), AC.

Reseda lutea subsp. neglecta (Müller Arg.) Ball.

Th., (O1-2-3, A1-2, K1-2-3, C1, H1-2, Hd, AS12-3, SS1-2 et SC), AC.

Reseda luteola $\mathrm{L}$. 
Th., (O1-2-3, A1-2, K1-2-3 et C1), AC.

Reseda phyteuma subsp. phyteuma

Th., (O1-2-3, A1-2, K1-2-3, C1, H1-2, Hd et AS1-2-3), R.

Sesamoides purpurascens (L.) G. Lopez

Ch., (O3), AC.

\section{ROSACEAE}

Crataegus monogyna Jacq.

Ph., (O1-2-3, A1-2, K1-2-3, C1 et AS1-2-3), C. Rosa canina $\mathrm{L}$.

Ph., (O1-2-3, A1-2, K1-2-3, C1 et AS3), C.

Rubus ulmifolius Schott.

Phl., (O1-2-3, A1-2, K1-2-3, C1 et AS3), C.

Sanguisorba minor subsp. alveolosa (Spach) Maire

H., (O1-2-3), C.

\section{RUBIACEAE}

Asperula hirsuta Desf.

Ch., (O1-2-3, A1-2, K1-2-3, C1, H1-2, Hd et AS1-2-3), [AfnI], C.

Rubia peregrina $\mathrm{L}$.

Phl., (O1-2-3, A1-2, K1-2-3, C1 et AS1-2-3), C.

\section{SALICACEAE}

Salix alba $\mathrm{L}$.

Ph., (O1-2-3, A1-2, K1-2-3, C1, H1-2, Hd et AS1-2-3), C.

\section{SAXIFRAGACEAE}

Saxifraga globulifera Desf.

Ch., (O1-2-3, A1-2, K1-2-3 et C1), [AMI], C.

Saxifraga carpetana var. atlantica (Boiss. \& Reut.)

Engl. \& Irmsch.

$$
\text { Ch., (O1-2-3, A1-2, K1-2-3, C1,AS1-2-3), R. }
$$

\section{SCROPHULARIACEAE}

Bartsia trixago L.

Th., (O1-2-3, A1-2, K1-2-3 et C1), C.

Linaria multicaulis subsp. heterophylla (Desf.)

D.A. Sutton

Th. (O3) [Afn], RR.

Linaria tristis subsp. marginata (Desf.) Maire

Th., (O3), [AM.], RR.
Misopates orontium (L.) Raf.

Th., (O1-2-3, A1-2, K1-2-3, C1, H1-2, Hd, AS12-3 et SC), C.

Parentucellia latifolia (L.) Caruel in Parl.

Th., (O1-2-3, A1-2, K1-2-3, C1, H1-2 et Hd), C. Scrophularia canina $\mathrm{L}$.

Ch., (O1-2-3, A1-2, K1-2-3, C1, H1-2, Hd et AS1-2-3), C.

\section{THYMELAECEAE}

\section{Daphne gnidium L.}

NPh., (O1-2-3, A1-2, K1-2-3 et C1), C.

\section{UMBELLIFERAE}

Ammoides pussilla (Bort.) Breistr.

Th., (O1-2-3, A1- 2, K1-2-3, C1, H1-2, Hd et AS1-2-3), C.

Daucus carota subsp. carota

Th. (H.), (O1-2-3, A1-2, K1-2-3 et C1), C.

Torilis arvensis subsp. recta Jury

Th., (O1-2-3, A1-2, K1-2-3, C1, H1-2, Hd et AS1-2-3), C.

\section{VALERIANACEAE}

Fedia cornucopiae (L.) Gaertn.

Th., (O1-2-3, A1-2, K1-2-3, C1, H1-2, Hd et AS1-2-3), [AMI], C.

Valerianella dentata (L.) Pollich

Th., (K1 et AS2), R.

\section{MONOCOTYLEDONES \\ ALLIACEAE}

Allium massaessylum Batt. et Trab.

G., (O3), [AM], RR.

\section{AMARYLLIDACEAE}

Narcissus cantabricus DC.

G., (O1-2-3 et AS2), [AMI], AC.

Narcissus tazetta L.

G., (O1-2-3), C.

\section{ASPARAGACEAE}

Asparagus acutifolius L.

Phl.(Ch.), (O1-2-3, A1-2, K1-2-3, C1 et AS1-2-3), C. 


\section{ASPHODELACEAE}

Asphodelus acaulis Desf.

G., (H2, A2 et O1-3), [Afn.], AC.

Asphodelus ramosus $\mathrm{L}$.

G., (A1, H1-2 et AS1-2-3), C.

\section{COLCHICACEAE}

Merendera filifolia Camb.

G., (O1-2-3, A1-2, K1-2-3 et C1), AC.

\section{DISCOREACEAE}

Tamus communis L.

G., (O1-2-3, A1-2, K1-2-3 et C1), C.

\section{HYACYNTHACEAE}

Charybdis maritima (L.) Speta

G., (O1-2-3, A1-2, K1-2-3 et C1), C.

Dipcadi serotinum (L.) Medik.

G., (O1-2-3, H1-2, Hd et AS1-2-3), C.

Muscari neglectum Gruss. Ex Ten.

G., (O1-2-3 et $\mathrm{C} 1), \mathrm{AC}$.

Muscari comosum (L.) Mill.

G., (O1-2-3, A1-2, K1-2-3, C1, H1-2, Hd et AS1-2-3), C.

Oncostema peruviana (L.) Speta

G., (O1-2-3, A1-2, K1-2-3, C1, H1-2, Hd et AS1-2-3), C.

Ornithogalum algeriense Jord. \& Fourr.

G., (O1- 2-3, A1-2, K1-2-3 et C1), [AfnI], C.

Prospero autumnale (L.) Speta

G., (O1-2-3, A1-2, K1-2-3, C1 et AS1-2-3), C.

Prospero fallax (Steinh.) Speta

G., (O1-2-3, A1-2, K1-2-3 et C1), [Afn.], AC.

\section{IRIDACEAE}

Gladiolus segetum Ker-Gawl.

G., (O1-2-3, A1-2, K1-2-3 et C1), C.

Iris tingitana Boiss. \& Reut.

G., (O1-2-3 et A1-2), [AM], AC

Romulea bulbocodium (L.) Sebast. \& Mauri

G., (O1-2-3, A1-2, K1-2-3, C1 et AS1-2-3), C.

\section{JUNCACEAE}

Juncus rigidus Desf.
Ch., (O1-2-3, A1-2, K1-2-3, C1, H1-2, Hd, AS12-3, SS1-2 et SC.), C.

\section{LILIACEAE}

Gagea algeriensis Chabert G., (O3 et A2), [AMI], R.

Gagea granatelli (Parl.) Parl. G., (O3, H1-2 et AS1-2-3), AC.

\section{ORCHIDACEAE}

Himantoglossum hircinum (L.) Spreng. G., (C1, A1, O3 et $\mathrm{H} 1), \mathrm{AC}$.

Ophrys atlantica Munby G., (O3), [AfnI], RR

Ophrys fusca Link. G., (O1-2-3, A1-2, K1-2-3 et C1), AC.

Ophrys tenthredinifera Willd.

G., (O1-2-3, A1-2, K1-2-3 et C1), C.

Orchis coriophora $\mathrm{L}$.

G., (O1-2-3, A1-2, K1-2-3 et C1), [AfnI], AC.

Orchis mascula (L.) L.

G., (O1-2-3, A1-2, K1-2-3, C1 et AS3), AC.

Orchis champagneuxii Barnéoud

G., (O1-3), AC.

\section{PALMAE}

Chamaerops humilis L.

NPh. (Ch.), (O1-2-3, A1-2, K1-2-3 et C1), C.

\section{POACEAE}

Aegilops neglecta Req. ex Bertol.

Th., (O1-2-3, A1-2, K1-2-3, C1, H1-2, Hd et AS1-2-3), C.

Ampelodesma mauritanica (Poir.) T. Durand\&

Schinz

H., (O1-2-3, A1-2, K1-2-3, C1 et AS2-3), C.

Aristida caerulescens Desf.

H., (O1-2-3, A1-2, C1, H1-2, Hd, AS1-2-3, SS12 et SC), AC.

Avena barbata Pott ex Link

Th., (O1-2-3, A1-2, K1-2-3, C1, H1-2, Hd et AS1-2-3), C.

Brachypodium distachyon (L.) P. Beauv.

Th., (O1-2-3, A1-2, K1-2-3, C1, H1-2, Hd, AS12-3 et SS1-2), C.

Briza minor L. 
Th., (O1-2-3, A1-2, K1-2-3 et C1), C. Bromus hodeaceus subsp. hordeaceus

Th., (O1-2-3, A1-2, K1-2-3, C1, H1-2, Hd, AS12-3 et SC), AC.

Bromus madritensis subsp. madritensis

Th., (O1-2-3, A1-2, K1-2-3 et C1), C.

Bromus rubens $\mathrm{L}$.

Th., (O1-2-3, A1-2, K1-2-3, C1, H1-2, Hd, AS12-3, SS1-2, SC), C.

Bromus tectorum $\mathrm{L}$.

Th., (O3, A2, K1-2-3, C1, H1-2, Hd, et AS12-3), C.

Bromus sterilis L.

Th., (K1-2, A2, AS1-2-3), AC.

Cynosurus echinatus L.

Th., (C1, A1-2 et O3), R.

Dactylis glomerata $\mathrm{L}$.

H., (O1-2-3, A1-2, K1-2-3, C1, H1-2, Hd, AS1-

2-3), C.

Desmazeria rigida (L.) Tutin

Th., (O1-2-3, A1-2, K1-2-3, C1, H1-2, Hd et AS1-2-3), C.

Festuca coerulescens Desf.

H., (O1-2-3, A1-2, K1-2-3, C1 et AS1-2-3), C. Lagurus ovatus L.

Th., (O1-2-3, A1-2, K1-2-3, C1, H1-2, Hd et AS1-2-3), C.

Lamarckia aurea (L.) Moench

Th., (O1-2-3, A1-2, K1-2-3 et C1), C.

Stipa tenacissima $\mathrm{L}$.

H., (O1-2-3, A2, C1, H1-2, Hd et AS1-2-3), AC.

\section{RUSCACEAE}

Ruscus hypophyllum $\mathrm{L}$.

H., (O1-2-3, A1-2, K1-2-3 et C1), C.

\section{SMILACACEAE}

Smilax aspera L.

Phl., (O1-2-3, A1-2, K1-2-3 et C1), C.

\section{DISCUSSION ET CONCLUSION}

L'inventaire des deux subéraies a permis de mettre en évidence 221 taxons de rang spécifique ou sous spécifique, répartis entre
65 familles, 164 genres. Les angiospermes dicotylédones forment le groupe systématique le plus important avec 161 taxons appartenant à 42 familles et 122 genres; les monocotylédones contiennent 50 unités taxonomiques réparties dans 15 familles et 38 genres. Les ptéridophytes et les gymnospermes comptent 8 familles, 9 genres et 11 taxons.

Les familles les plus riches sont les Asteraceae, Leguminosae, Poaceae, Lamiaceae, Cistaceae et Hyacinthaceae avec respectivement $25,22,18,13$ et 10 taxons chacune. Ces 5 familles détiennent presque $50 \%$ de la richesse totale du site.

Il n'y a qu'un taxon endémique algérien. Il s'agit de Coleostephus multicaulis. Les endémiques algéro-marocains comptent 7 taxons. Parmi eux, deux appartiennent aux Lamiaceae. Les familles des Brassicaceae, Leguminoseae, Scrophulariaceae, Alliaceae et Iridaceae sont représentées chacune par un seul taxon. Les taxons endémiques ibéroalgéro-marocains sont au nombre de 11 dont 4 rares à l'échelle nationale. Les Cistacaeae sont les plus représentées avec 3 taxons. Les Aristolochiaceae, Brassicaceae, Labiatae, Leguminosae, Saxifragaceae, Valerianaceae, Amaryllidaceae et Liliaceae comptent un taxon chacun. Les endémiques d'Afrique du Nord et les endémiques communes à la Péninsule ibérique et l'Afrique du nord sont représentées par 4 et 7 taxons respectivement.

L'importance des endémiques algéroibéro-marocains confirme l'appartenance des monts de Tlemcen au hotspot Bético-rifain (partagé entre la péninsule Ibérique, le Maroc et l'Algérie). Les endémiques algéro-marocains et ibéro-africains du nord représentent les taux les plus importants après la catégorie précitée. Les taxons endémiques algériens sont très peu nombreux. Les taxons rares non endémiques comptent 17 taxons répartis entre 13 familles. Les Leguminosae et les Asteraceae sont représentées chacune par 3 taxons, les autres par 1 taxon. 9 taxons sont endémiques et menacés. 
Ils sont répartis entre 8 familles.

Les taxons rares sont peu nombreux. La plupart des espèces inventoriées dans cette étude sont communes ou assez communes pour l'ensemble du territoire national.

$\mathrm{Si}$ on tient compte de l'ensemble des résultats de cet inventaire floristique, on ne peut que comfirmer l'importance de la dégradation et de la banalisation des paysages par l'installation d'espèces opportunistes favorisées par le pâturage (Anacyclus clavatus, Calendula arvensis, Carduus pycnocephalus, Echinops strigosus, Cynoglossum cheirifolium, Marrubium vulgare, Malva sylvestris, Papaver rhoeas, Plantago cupanii, Plantago lagopus, Rumex bucephalophorus, Anagallis arvensis subsp. latifolia, Reseda alba Daphne gnidium, Asphodelus ramosus et Charybdis maritima...). La dominance des thérophytes par rapport aux autres types biologiques est tout a fait révélatrice. Même si le pourcentage des thérophytes est habituellement élevé dans les formations végétales méditerranéennes, avec l'aridité (Daget, 1980) et la dégradation (Grime 1977), ce pourcentage augmente d'une manière très significative dominant largement les autres types biologiques comme pour notre cas présent.

Pour le moment des lambaux forestiers se mantiennent, malgré l'ampleur de la dégradation. Des efforts urgents de protection doivent être entrepris afin de préserver les deux espèces forestières à notre avis les plus intéressantes dans le parc. Il s'agit bien, du chêne liège et du chêne zéen avec les espèces endémiques qui leurs sont inféodées. Ces dernières constituent une véritable liste rouge, avec notamment:

-Hieracium amplexicaule: espèce rarissime signalée par Quézel et Santa (1962) au niveau Djebel Touggour, retrouvée dans la mise en défens installée par la direction du parc au niveau de la forêt de Zariffet en 2008. Espèce protégée par la loi de 1993. -Eruca setulosa: endémique algéromarocaine (considérée au départ par Quézel et Santa comme endémique algérienne mais découvert au Maroc) rarissime, signalée uniquement dans la région de Ghar Rouban. Découverte dans les mises en défens de Zariffet en 2008. Espèce protégée par la loi de 1993. -Halimium umbellatum subsp. viscosum: Endémique algéro-ibéro-marocaine; en Algérie, elle est présente uniquement dans la région de Hafir et Zariffet. -Helianthemum origanifolium subsp. molle : Endémique algéro-ibéro-marocaine présente dans la région littorale oranaise. Découverte dans la forêt de Hafir en 2004.

-Quercus faginea subsp.tlemcenensis: Endémique algéro-marocaine. Espèce assez commune mais sa préservation permet le maintient de beaucoup d'autres. -Quercus suber: la préservation du chêne liège est l'une des principales tâches du parc national de Tlemcen. -Ulex boivinii: endémique algéro-ibéromarocaine rare présente seulement au niveau des monts Tlemcen. -Linaria multicaulis subsp. heterophylla endémique d'Afrique du Nord, rarissime -Linaria tristis subsp. marginata endémique algéro-marocainelocaliséeauniveau desmonts de Tlemcen dans la région de Ghar Rouban, découverte dans la forêt de Hafir en 2006. -Allium massaessylum endémique algéroibéro-marocaine très rare signalée au niveau de la forêt de Hafir par Quezel et Santa (1962) jamais revue depuis. -Gagea algeriensis endémique ibéroalgéro-marocaine rare, localisée dans le Tell algérois et le Tell oranais. -Ophrys atlantica orchidée ibéronord africaines très rare, signalée dans la région mais jamais redécouverte.

\section{BIBLIOGRAPHIE}

DAHMANI, M. -1984- Contribution à l'étude des groupements à chêne vert (Quercus rotundifolia Lamk) des monts de Tlemcen (ouest algérien). Approche phytocoenologique et 
phytosociologique. Thèse 3ème cycle USTHB, Alger. $238 \mathrm{p}$.

DAGET, Ph. -1980- Sur les types biologiques botaniques en tant que stratégies adaptatives (cas des Thérophytes). Actes du Colloque D'Ecologie théoriques, E.N.S, Paris: 89-114.

FENNANE, M., M. IBN TATTOU, M. J. MATHEZ, A. OUYAHYA, \& J. El OUALIDI (Ed.) -1999Flore pratique du Maroc. Vol. I. Trav. Inst. Sci., sér. Bot. 36, Rabat.

FENNANE, M., M. IBN TATTOU, A. OUYAHYA, \& EL OUALIDI J. (Ed.) -2007- Flore pratique du Maroc. Vol II, Trav. Inst. Sci., sér. Bot. 38, Rabat.

GAOUAR, A. -1980- Hypothèse et réflexion sur la dégradation des écosystèmes forestiers dans la région de Tlemcen. Revue Forêt méditerranéenne, Tome 2: 131-146.

GRIME J.P. -1977- Evidence for the existence of three primary strategies in plants and its relevance to ecological and evolutionary theory. American Naturalist 111: 1169-1194.

LETREUCH-BELAROUCI, N. -1991- Les reboisements en Algérie et leur perspective d'avenir. Vol. I. OPU, Alger. 294 p.
LETREUCH-BELAROUCI，A. -2002Compréhension du processus de dégradation de la subéraie de Tlemcen et possibilités d'installation d'une réserve forestière. Thèse de Magistère. Université de Tlemcen, Algérie. 205p.

PARC NATIONAL DE TLEMCEN - 2000- La subéraie de Hafir Tlemcen (Oranie Algérie). Rapport du Parc National de Tlemcen. 7p.

QUEZEL, P. \& S. SANTA -1962-1963. Nouvelle flore de l'Algérie et des régions désertiques méridionales. Tome I et Tome II. CNRS, Paris, $1087 \mathrm{p}$.

VALDÉS, B., M. REJDALI, A. A. E. KADMIRI, S. L. JURY \& J. M. MONTSERRAT -2002Catalogue des plantes vasculaires du Nord du Maroc incluant des clés d'ídentification. Vol. I \& II, Consejo Superior de Investigaciones Científicas, Madrid. 1007p.

ZERAIA, A. -1981- Essais d'interprétation comparative des données écologiques, phénologiques et de production subéro-ligneuse dans les forêts de chêne liège de provenance cristalline (France méridionale) et Algérie. Thèse Doctorat Université Aix-Marsaille. 367 p. 
